Received: 31.08 .2018

Revised: 27.09 .2018

Accepted: 19.10 .2018

DOI: $10.17804 / 2410-9908.2018 .5 .064-073$

\title{
A PROCEDURE FOR STUDYING THE EFFECT OF RADIATION DEFECTS ON THE DIFFUSION COEFFICIENTS OF DEUTERIUM IN METALS
}

\author{
O. A. Nefedova ${ }^{1, a)^{*}}$, V. B. Vykhodets ${ }^{2}$, and T. E. Kurennykh ${ }^{2}$ \\ ${ }^{I}$ Institute of Engineering Science, Ural Branch of the Russian Academy of Sciences, \\ 34 Komsomolskaya St., Ekaterinburg, Russian Federation \\ ${ }^{2}$ M.N. Miheev Institute of Metal Physics, Ural Branch of the Russian Academy of Sciences, \\ 18 S. Kovalevskoy St., Ekaterinburg, Russian Federation \\ a) iD https://orcid.org/0000-0003-1163-6699, , nefedova@imach.uran.ru; \\ *Corresponding author. E-mail: nefedova@imach.uran.ru \\ Address for correspondence: ul. Komsomolskaya, 34, Ekaterinburg, 620049, Russian Federation \\ Tel.: +7 (343) 3623022
}

The paper proposes and examines an approach to the investigation of the effect of radiation defects on the diffusion coefficients of deuterium in metals with the application of the online nuclear reaction analysis technique (NRAOL). In the development of the method, an assumption was used that the diffusion system is described by two different values of the diffusion coefficients of deuterium, corresponding to the irradiated and unirradiated regions of the sample. The diffusion boundary value problem is formulated and the analytical expression for the calculation of deuterium concentration in the sample is obtained. The simulation calculations are carried out; the depth and time dependences of deuterium concentration in the sample are constructed. A computational algorithm based on the least squares method is developed in order to investigate the effect of radiation defects on the diffusion coefficients of deuterium by NRAOL. Numerical calculations are performed for samples of nickel and sodium by an algorithm-based computer program.

Keywords: deuterium diffusion, metals, nuclear reaction analysis, radiation defects, diffusion boundary value problem, analytical solution.

\section{Acknowledgment}

The work was performed within the state assignment from FASO Russia (theme Spin, No. 01201463330) and partially supported by RFBR (project No. 17-03-00101).

\section{References}

1. Ishikava T., Mclellan R.B. The low-temperature diffusion of Hydrogen through annealed, quenched and aged gold. Journal of Physics and Chemistry of Solids, 1985, vol. 46, iss. 12, pp. 1393-1396. DOI: 10.1016/0022-3697(85)90078-2.

2. Ishikava T., Mclellan R.B. The G.P. zones in Al-Cu alloys-I. Acta Metallurgica, 1985, vol. 33, iss. 11, pp. 1945-1955. DOI: 10.1016/0001-6160(85)90117-8.

3. Vykhodets V.B., Kurennykh T.E., Nefedova O.A., Gorelov V.P., Stroeva A.Yu., Balakireva V.B., Vykhodets E.V., Obukhov S.I. Deuterium diffusion in proton conductors $\mathrm{La}_{0.9} \mathrm{Sr}_{0.1} \mathrm{ScO}_{3-\delta}$ and $\mathrm{BaZr}_{0.9} \mathrm{Y}_{0.1} \mathrm{O}_{3-\delta}$ at room temperature. Solid State Ionics, 2014, vol. 263, pp. 152-156. DOI: $10.1016 /$ j.ssi.2014.06.003.

4. $\quad$ Vykhodets V.B., Kurennykh T.E., Nefedova O.A. Application of Nuclear Reaction Analysis to Studying Hydrogen Diffusion and Nonstoichiometry in Metal Oxides. Diagnostics, Resource and Mechanics of materials and structures, 2015, iss. 5, pp. 31-44. Available at: http://dreamjournal.org/issues/2015-5/2015-5_57.html 
5. Lykov A.V. Teoriya teploprovodnosti [Heat Conduction Theory]. M., Vysshaya Shkola Publ., 1967, 600 p. (In Russian).

6. Carslaw H.S., Jaeger J.C. Conduction of Heat in Solids. London, Oxford University Press Publ., 1959, 510 p. ISBN-10: 0198533039, ISBN-13: 978-0198533030.

7. G. Doetsch. Anleitung zum praktischen Gebrauch der Laplace-Transformation und der Z-Transformation. München, Wien, Oldenbourg Publ., 1989, 256 p. ISBN-10: 3486213105, ISBN-13: 9783486213102.

8. $\quad$ Lobanov M.L., Zorina M.A. Metody opredelenia koeffitsientov diffuzii [Diffusion Coefficient Determination Methods]. Ekaterinburg, Ural University Publ, 2017, 100 p. (In Russian). 
Подана в журнал: 31.08 .2018

УДК 544.6:544.16:517.958

DOI: $10.17804 / 2410-9908.2018 .5 .064-073$

\title{
МЕТОДИКА ДЛЯ ИССЛЕДОВАНИЯ ВЛИЯНИЯ РАДИАЦИОННЫХ ДЕФЕКТОВ НА КОЭФФИЦИЕНТЫ ДИФФУЗИИ ДЕЙТЕРИЯ В МЕТАЛЛАХ
}

\author{
О. А. Нефедова ${ }^{1,)^{*}}$, В. Б. Выходец, б), Т. Е. Куренных ${ }^{2, \text { в) }}$ \\ ${ }^{I}$ Федеральное государственное бюджетное учреждение науки Институт машиноведения \\ Уральского отделения Российской академии наук, ул. Комсомольская, 34, Екатеринбург, Российская Федераиия \\ ${ }^{2}$ Федеральное государственное бюджетное учреждение науки Институт физики металлов им. М.Н. Михеева \\ Уральского отделения Российской академии наук, ул. С. Ковалевской, 18, Екатеринбург, Российская Федерация \\ a) (iD https://orcid.org/0000-0003-1163-6699, ㅈefedova@imach.uran.ru; \\ * Ответственный автор. Электронная почта: nefedova@imach.uran.ru \\ Адрес для переписки: ул. Комсомольская, 34, Екатеринбург, Российская Федерация \\ Тел.: +7 (343) 362-30-22
}

В работе предложен и апробирован подход для исследовании влияния радиационных дефектов на коэффициенты диффузии дейтерия в металлах с использованием методики ядерных реакций в режиме он-лайн (NRAOL). При разработке метода было использовано предположение о том, что диффузионная система описывается двумя отличающимися друг от друга значениями коэффициентов диффузии дейтерия, которые соответствуют облучаемой и необлучаемой зонам образца. Сформулирована краевая задача диффузии и получено аналитическое выражение для расчета концентрации дейтерия в образце. Выполнены модельные расчеты и построены зависимости концентрации дейтерия от глубины в образце и от времени. На основе метода наименьших квадратов разработан вычислительный алгоритм для исследования влияния радиационных дефектов на коэффициенты диффузии дейтерия в рамках методики NRAOL. Для образцов никеля и натрия проведены численные расчеты с помощью компьютерной программы, созданной на базе алгоритма.

Ключевые слова: диффузия дейтерия, металлы, ядерный микроанализ, радиационные дефекты, краевая задача диффузии, аналитическое решение.

\section{1. Введение}

Предметом работы является разработка методики для исследования влияния точечных дефектов на коэффициенты диффузии $D$ водорода в металлах. Эта задача представляет большой интерес для теории диффузии и практических приложений, поскольку водород участвует во многих технологических процессах и часто необходимы данные по диффузии водорода в металлах, подвергнутых пластической деформации, радиационному облучению и термической обработке. В литературе большинство данных по этому вопросу получено, когда были разработаны методики для измерения коэффициентов диффузии водорода, при применении которых отжиги образцов проводились не в газообразном водороде, а в жидких электролитах. Такие методики позволяли проводить измерения $D$ при низких по диффузионным масштабам температурах, они составляли от единиц до нескольких десятков ${ }^{\circ} \mathrm{C}$. С помощью электролитической методики в работе [1] были выполнены измерения $D$ в интервале температур от 5 до $60{ }^{\circ} \mathrm{C}$ в отожженных образцах золота и закаленных с высоких температур, в закаленных образцах значения $D$ были в 5-6 раз ниже, чем в отожженных. Эти результаты получили простую трактовку: в закаленных образцах содержатся точечные дефекты, в частности вакансии, они служили ловушками для атомов водорода, что приводило к уменьшению значений $D$. Аналогичные исследования проводились также в платине, меди и серебре [2]. В целом в перечисленных работах было констатировано, что использованный 
подход не привел к получению однозначной информации: результаты различных работ противоречили друг другу, в том числе были получены данные, свидетельствующие о том, что в пределах погрешности экспериментов не наблюдалось влияния точечных дефектов на коэффициенты диффузии водорода в металлах.

В настоящей работе предлагается альтернативный подход для исследования этого эффекта. Он основан на применении методики ядерных реакций в режиме он-лайн для исследования диффузии дейтерия в материалах [3]. При использовании NRAOL производится непрерывное облучение образцов ускоренными дейтронами и в режиме реального времени осуществляется наблюдение за диффузией имплантированных атомов дейтерия. Отметим два обстоятельства, по которым методика NRAOL является перспективной для исследования влияния точечных дефектов на коэффициенты диффузии дейтерия: во-первых, при функционировании NRAOL в образце создаются точечные радиационные дефекты и, во-вторых, эта методика применима для исследования диффузии в металлах при очень низких температурах, в том числе криогенных. Последнее обстоятельство представляется принципиальным, так как во многих металлах точечные дефекты отжигаются в районе комнатной температуры.

\section{2. Постановка краевой задачи диффузии}

Рассмотрим одномерную краевую задачу диффузии дейтерия в полуограниченном образце $0<x<\infty$. При применении методики NRAOL диффузия атомов дейтерия в образце протекает из бесконечно тонкого слоя с глубиной залегания $x=x_{0}$, на которой ускоренные дейтроны полностью теряют свою кинетическую энергию. Диффузия осуществляется к левой (облучаемой) и правой (необлучаемой) границам образца, это происходит при непрерывном функционировании источника постоянной мощности, расположенного на глубине $x=x_{0}$. Будем полагать, что радиационные дефекты, оказывающие влияние на коэффициенты диффузии, существуют только в зоне образца, расположенной слева от источника диффузии. Дополнительно постулируем, что диффузионная система описывается всего двумя отличающимися друг от друга значениями коэффициентов диффузии дейтерия: $D_{1}$ и $D_{2}$, которые соответствуют облучаемой $\left(0<x<x_{0}\right)$ и необлучаемой $\left(x_{0}<x<\infty\right)$ зонам образца. Каждый из этих коэффициентов диффузии будем считать не зависящим от координаты $x$ и времени $t$ облучения образца. Соответствующие концентрационные профили атомов дейтерия в левой и правой зонах образца обозначим $c_{1}(x, t)$ и $c_{2}(x, t)$. Для получения данных о влиянии радиационных дефектов на коэффициенты диффузии дейтерия требуется найти решение следующей системы уравнений диффузии:

$$
\left\{\begin{array}{l}
\frac{\partial c_{1}(x, t)}{\partial t}=D_{1} \frac{\partial^{2} c_{1}(x, t)}{\partial x^{2}}, t>0,0<x<x_{0}, \\
\frac{\partial c_{2}(x, t)}{\partial t}=D_{2} \frac{\partial^{2} c_{2}(x, t)}{\partial x^{2}}+f(x, t), t>0, x_{0}<x<\infty .
\end{array}\right.
$$

Здесь функция $f(x, t)=w \delta\left(x-\left(x_{0}+\varepsilon\right)\right)$ описывает работу источника постоянной мощности $w ; \delta\left(x-\left(x_{0}+\varepsilon\right)\right)$ - дельта-функция Дирака; $\varepsilon>0$ - бесконечно малая величина.

Начальное условие задачи предполагает отсутствие дейтерия в образце до облучения

$$
c_{1}(x, 0)=c_{2}(x, 0)=0 .
$$

Граничные условия запишем в виде:

$$
\begin{gathered}
\left.\frac{\partial c_{1}(x, t)}{\partial x}\right|_{x=0}=0 \\
c_{2}(\infty, t)=0
\end{gathered}
$$




$$
c_{1}\left(x_{0}, t\right)=c_{2}\left(x_{0}, t\right),\left.D_{1} \frac{\partial c_{1}(x, t)}{\partial x}\right|_{x=x_{0}}=\left.D_{2} \frac{\partial c_{2}(x, t)}{\partial x}\right|_{x=x_{0}},
$$

они соответствуют отражающему свойству левой границы образца, значительной удаленности от источника правой границы образца, а также равенству концентраций и потоков ионов дейтерия по обе стороны от границы раздела двух областей.

Аналитические выражения для концентрационных профилей $c_{1}(x, t)$ и $c_{2}(x, t)$ задачи (1)-(5) найдем, используя метод интегрального преобразования Лапласа [4].

\section{3. Решение краевой задачи диффузии}

Применим преобразования Лапласа относительно переменной $t \quad c_{L i}(x, s)=$ $=\int_{0}^{\infty} c_{i}(x, t) \exp (-s t) d t(i=1,2)$ к системе уравнений $(1)$, т. е. умножим правые и левые части уравнений системы на $\exp (-s t)$ и проинтегрируем по $t$ в пределах от нуля до бесконечности. В результате вместо системы дифференциальных уравнений в частных производных относительно оригиналов функций $c_{1}(x, t)$ и $c_{2}(x, t)$ получим вспомогательную систему обыкновенных дифференциальных уравнений для изображений функций $c_{L 1}(x, s)$ и $c_{L 2}(x, s)$ :

$$
\left\{\begin{array}{c}
\frac{d^{2} c_{L 1}(x, s)}{d x^{2}}-\frac{s}{D_{1}} c_{L 1}(x, s)=0, t>0,0<x<x_{0}, \\
\frac{d^{2} c_{L 2}(x, s)}{d x^{2}}-\frac{s}{D_{2}} c_{L 2}(x, s)+\frac{w}{D_{2} s} \delta\left(x-\left(x_{0}+\varepsilon\right)\right)=0, t>0, x_{0}<x<\infty, \varepsilon \rightarrow+0 .
\end{array}\right.
$$

Здесь переменная $s$ играет роль параметра, от которого зависит решение.

Преобразуем аналогичным образом начальное (2) и граничные (3)-(5) условия задачи и получим краевые условия для изображений:

$$
\begin{gathered}
c_{L 1}(x, 0)=c_{L 2}(x, 0)=0 ; \\
\left.\frac{d c_{L 1}(0, s)}{d x}\right|_{x=0}=0 ; \\
c_{L 2}(\infty, s)=0 ; \\
c_{L 1}\left(x_{0}, s\right)=c_{L 2}\left(x_{0}, s\right),\left.D_{1} \frac{d c_{L 1}(x, s)}{d x}\right|_{x=x_{0}}=\left.D_{2} \frac{d c_{L 2}(x, s)}{d x}\right|_{x=x_{0}} .
\end{gathered}
$$

Решение задачи (6) для изображений функций при условиях (7) и (9) имеет вид:

$$
\begin{gathered}
c_{L 1}(x, s)=A \exp \left(\sqrt{\frac{s}{D_{1}}} x\right)+B \exp \left(-\sqrt{\frac{s}{D_{1}}} x\right) ; \\
c_{L 2}(x, s)=C \exp \left(-\sqrt{\frac{s}{D_{2}}} x\right)+\hat{c}_{L 2}(x, s) .
\end{gathered}
$$

Здесь $A, B, C$ - постоянные, зависящие от параметра $s ; \hat{c}_{L 2}(x, s)$ - частное решение неоднородного уравнения системы (6), определяемое выражением 


$$
\hat{c}_{L 2}(x, s)=\frac{w}{2 s \sqrt{s D_{2}}} \theta\left(\left(x_{0}+\varepsilon\right)-x\right)\left(\exp \left(2 \sqrt{\frac{s}{D_{2}}}\left(x-x_{0}\right)\right)-1\right) \exp \left(-\sqrt{\frac{s}{D_{2}}}\left(x-x_{0}\right)\right)
$$

где $\theta(y)=\left\{\begin{array}{l}0, y<0, \\ 1, y \geq 0 .\end{array}-\right.$ функция Хевисайда.

Постоянные $A, B, C$ найдем из граничных условий (8) и(10). После нахождения и подстановки выражений для $A, B, C$ в выражения (11), (12) устремим $\varepsilon$ к нулю и учтем, что $\lim _{\varepsilon \rightarrow 0} \hat{c}_{L 2}(x, s)=0$, так как $\theta\left(x_{0}-x\right)=0$ при $x>x_{0}$. В результате получим решение вспомогательной системы уравнений

$$
\begin{gathered}
c_{L 1}(x, s)=\frac{w}{s \sqrt{s}} \frac{\left[\exp \left(\sqrt{\frac{s}{D_{1}}}\left(x_{0}+x\right)\right)+\exp \left(\sqrt{\frac{s}{D_{1}}}\left(x_{0}-x\right)\right]\right]}{\left[\left(\sqrt{D_{1}}+\sqrt{D_{2}}\right) \exp \left(2 \sqrt{\frac{s}{D_{1}}} x_{0}\right)+\left(\sqrt{D_{2}}-\sqrt{D_{1}}\right)\right]} \\
c_{L 2}(x, s)=\frac{w}{s \sqrt{s}} \frac{\exp \left(\sqrt{\frac{s}{D_{2}}}\left(x_{0}-x\right)\left(1+\exp \left(2 \sqrt{\frac{s}{D_{1}}} x_{0}\right)\right)\right]}{\left[\left(\sqrt{D_{1}}+\sqrt{D_{2}}\right) \exp \left(2 \sqrt{\frac{s}{D_{1}}} x_{0}\right)+\left(\sqrt{D_{2}}-\sqrt{D_{1}}\right)\right]} .
\end{gathered}
$$

Для того чтобы записать решение исходной задачи (1)-(5), необходимо выполнить обратное преобразование Лапласа, т. е. перейти от изображений функций к их оригиналам с помощью специальной таблицы изображений из [4]. Следовательно, решение в изображениях необходимо переписать в виде, соответствующем табличному. Для этого умножим числители и знаменатели соотношений (13) и (14) на выражение $\left(\sqrt{D_{1}}+\sqrt{D_{2}}\right) \exp \left(-2 x_{0} \sqrt{s / D_{1}}\right)$ и положим $h=\left(\sqrt{D_{1}}-\sqrt{D_{2}}\right) /\left(\sqrt{D_{1}}+\sqrt{D_{2}}\right)$. Так как $|h|<1$, то проведем преобразование на основе разложения

$$
\frac{1}{1-z}=\sum_{n=0}^{\infty} z^{n}=1+z+z^{2}+\ldots, \text { если }|z|<1
$$

и получим:

$$
\begin{gathered}
\left.c_{L 1}(x, s)=\frac{w}{s \sqrt{s}\left(\sqrt{D_{1}}+\sqrt{D_{2}}\right.}\right) \sum_{n=0}^{\infty} h^{n}\left[\exp \left(-\sqrt{s} \frac{x_{0}(1+2 n)-x}{\sqrt{D_{1}}}\right)+\exp \left(-\sqrt{s} \frac{x_{0}(1+2 n)+x}{\sqrt{D_{1}}}\right)\right], \\
\left.c_{L 2}(x, s)=\frac{w}{s \sqrt{s}\left(\sqrt{D_{1}}+\sqrt{D_{2}}\right.}\right)_{n=0}^{\infty} h^{n}\left[\exp \left(-\sqrt{s}\left(\frac{x-x_{0}}{\sqrt{D_{2}}}+\frac{2 x_{0}(1+n)}{\sqrt{D_{1}}}\right)\right)+\exp \left(-\sqrt{s}\left(\frac{x-x_{0}}{\sqrt{D_{2}}}+\frac{2 x_{0} n}{\sqrt{D_{1}}}\right)\right)\right] .
\end{gathered}
$$

Далее из таблицы изображений найдем соответствующие оригиналы функций и запишем решение задачи (1)-(5): 


$$
\begin{aligned}
& \left.c_{1}=\frac{w}{\left(\sqrt{D_{1}}+\sqrt{D_{2}}\right.}\right) \sum_{n=0}^{\infty} h^{n}\left[2 \sqrt{\frac{t}{\pi}}\left(\exp \left(-\frac{k_{1}^{2}}{4 t}\right)+\exp \left(-\frac{k_{2}^{2}}{4 t}\right)\right)-k_{1} \operatorname{erfc}\left(\frac{k_{1}}{2 \sqrt{t}}\right)-k_{2} \operatorname{erfc}\left(\frac{k_{2}}{2 \sqrt{t}}\right)\right], \\
& c_{2}=\frac{w}{\left(\sqrt{D_{1}}+\sqrt{D_{2}}\right)} \sum_{n=0}^{\infty} h^{n}\left[2 \sqrt{\frac{t}{\pi}}\left(\exp \left(-\frac{k_{3}^{2}}{4 t}\right)+\exp \left(-\frac{k_{4}^{2}}{4 t}\right)\right)-k_{3} \operatorname{erfc}\left(\frac{k_{3}}{2 \sqrt{t}}\right)-k_{4} \operatorname{erfc}\left(\frac{k_{4}}{2 \sqrt{t}}\right)\right],
\end{aligned}
$$

где $k_{1}=\left(x_{0}(1+2 n)-x\right) / \sqrt{D_{1}} ; \quad k_{2}=\left(x_{0}(1+2 n)+x\right) / \sqrt{D_{1}} ; \quad k_{3}=\left(x-x_{0}\right) / \sqrt{D_{2}}+2 x_{0}(1+n) / \sqrt{D_{1}}$; $k_{4}=\left(x-x_{0}\right) / \sqrt{D_{2}}+2 x_{0} n / \sqrt{D_{1}}$.

Аналитические выражения (15) и (16) были далее использованы при решении обратной задачи по определению независимых коэффициентов диффузии $D_{1}$ и $D_{2}$. При использовании методики NRAOL измерение концентрационных профилей возможно только в левой (облучаемой) зоне образца, и поэтому в работе был разработан метод расчета коэффициентов $D_{1}$ и $D_{2}$, основанный на том, что профили в облучаемой и необлучаемой зонах образца связаны друг с другом через граничные условия (5) и общий источник диффузии, расположенный на границе соприкосновения.

\section{4. Модельные расчеты}

Для иллюстрации возможностей методики NRAOL выполним модельные расчеты с помощью выражений (15) и (16) зависимостей концентрации дейтерия от глубины в образце и от времени. Значения физических характеристик, используемых в расчетах, соответствовали экспериментальному режиму методики NRAOL для образцов никеля. B соответствии с литературными данными в модельных расчетах принималось, что точечные дефекты приводят к уменьшению коэффициентов диффузии, т.е. $D_{1}<D_{2}$.

На рис. 1 представлены зависимости концентрации дейтерия от глубины в образце $c\left(x, t_{k}\right)(k=1,2,3)$ в фиксированные моменты времени: а) $t_{1}=180 \mathrm{c} ;$ б) $t_{2}=3600 \mathrm{c} ;$ в) $t_{3}=10000 \mathrm{c}$. Расчеты были выполнены при следующих значениях параметров: $w=10^{18}\left(\mathrm{~m}^{2} \cdot \mathrm{c}\right)^{-1} ; D_{1}=10^{-14} \mathrm{~m}^{2} / \mathrm{c}$; $D_{2}=10^{-12} \mathrm{M}^{2} / \mathrm{c} ; x_{0}=3,15$ мкм. Из результатов модельных расчетов, следует, что расчетные зависимости концентрации дейтерия от глубины в образце, для фиксированного момента времени $t_{k}$, обнаруживают максимумы на глубине, равной глубине расположения источника. Кроме того, на всех глубинах концентрации дейтерия возрастали при увеличении времени $t_{k}$ облучения образца (рис. 1).

На рис. 2, 3 и 4 приведены временные зависимости $c\left(x^{*}, t\right)$ на фиксированных глубинах $x^{*}$ для различных значений пары коэффициентов диффузии $D_{1}$ и $D_{2}$. Для рис. 2 была выбрана глубина вблизи поверхности $x^{*}=0,15$ мкм, для рис. 3 - вблизи источника, в необлучаемой области $x^{*}=3$ мкм, а для рис. 4 - вблизи источника, в облучаемой области $x^{*}=3,3$ мкм. При моделировании принимали следующие значения параметров: $w=10^{18}\left(\mathrm{M}^{2} \cdot \mathrm{c}\right)^{-1} ; x_{0}=3,15$ мкм. Расчетные данные, представленные на рисунках, показывают, что концентрация дейтерия на всех глубинах монотонно возрастает при увеличении времени. При этом вблизи поверхности, на глубине $x^{*}=0,15$ мкм, кривые $c\left(x^{*}, t\right)$ характеризуются увеличением производной $d c\left(x^{*}, t\right) / d t$ при возрастании времени для низких значений коэффициентов диффузии $10^{-16} \leq D_{1} \leq 10^{-15}, 10^{-15} \leq D_{2} \leq 10^{-14}$ и уменьшением производной $d c\left(x^{*}, t\right) / d t$ для более высоких значений $D_{1}=10^{-14} \mathrm{~m}^{2} / \mathrm{c} ; D_{2}=10^{-13} \mathrm{~m}^{2} / \mathrm{c}$. Вблизи источника, с обеих сторон, наблюдается уменьшение производной $d c\left(x^{*}, t\right) / d t$ при увеличении времени $t$ облучения образцов для всех коэффициентов диффузии из интервала $10^{-16} \mathrm{~m}^{2} / \mathrm{c} \leq D_{1} \leq 10^{-14} \mathrm{~m}^{2} / \mathrm{c} ; 10^{-15} \mathrm{~m}^{2} / \mathrm{c} \leq D_{2} \leq 10^{-13} \mathrm{~m}^{2} / \mathrm{c}$. 


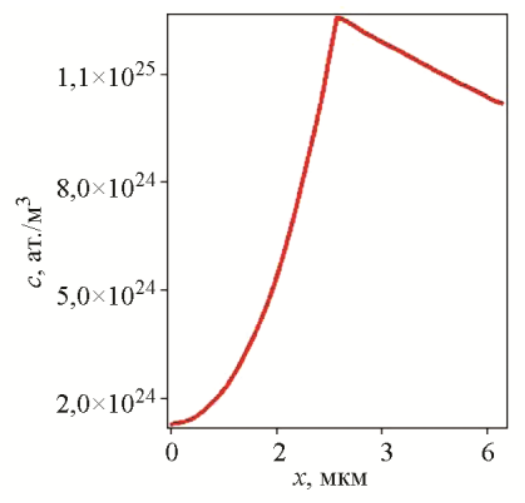

$a$

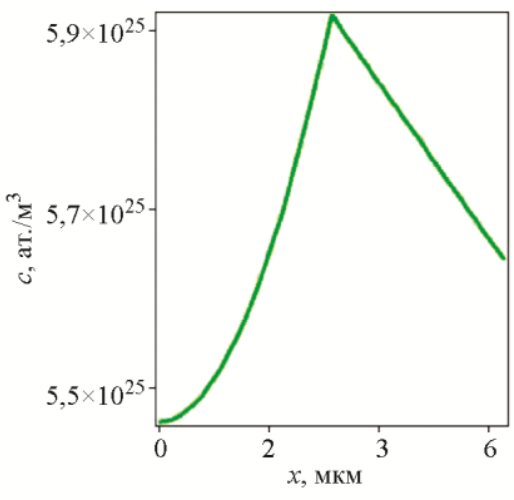

б

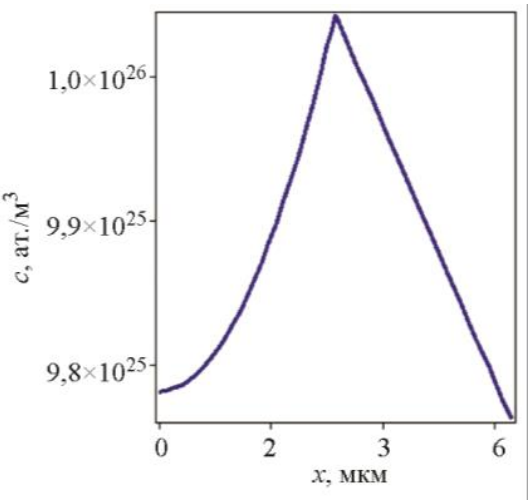

B

Рис. 1. Расчетные зависимости концентрации дейтерия от глубины в образце в заданные моменты времени: $a-t_{1}=180 \mathrm{c} ; \sigma-t_{2}=3600 \mathrm{c} ; \boldsymbol{s}-t_{3}=10000 \mathrm{c}$.

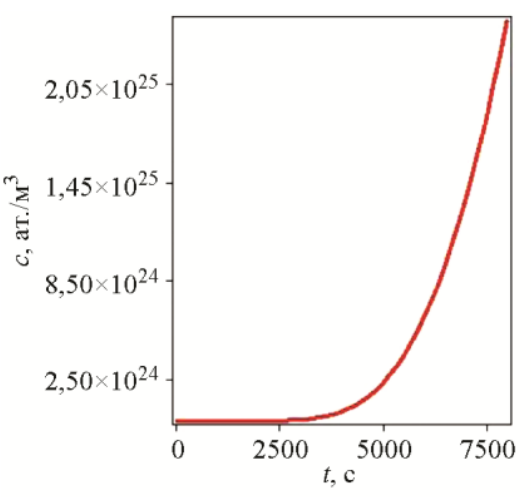

$a$

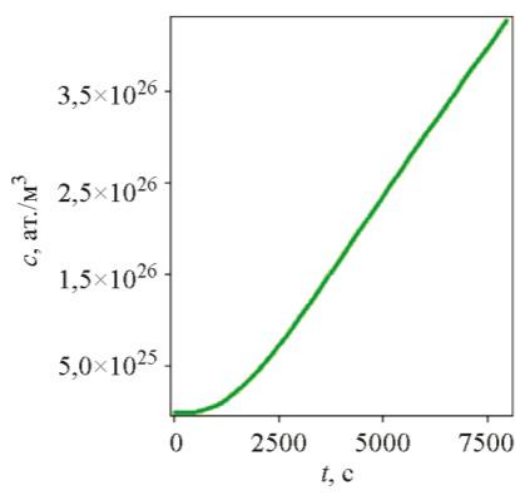

$\sigma$

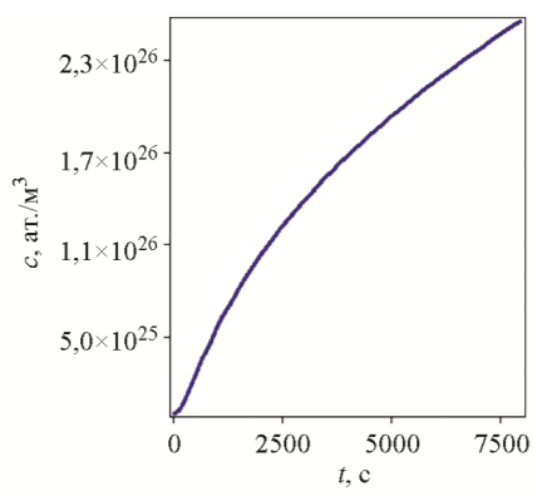

B

Рис. 2. Расчетные зависимости концентрации дейтерия от времени на глубине 0,15 мкм для различных значений пары коэффициентов диффузии $D_{1}$ и $D_{2}: a-D_{1}=10^{-16} \mathrm{~m}^{2} / \mathrm{c}$; $D_{2}=10^{-15} \mathrm{M}^{2} / \mathrm{c} ; \sigma-D_{1}=10^{-15} \mathrm{~m}^{2} / \mathrm{c} ; D_{2}=10^{-14} \mathrm{M}^{2} / \mathrm{c} ; \theta-D_{1}=10^{-14} \mathrm{M}^{2} / \mathrm{c} ; D_{2}=10^{-13} \mathrm{M}^{2} / \mathrm{c}$

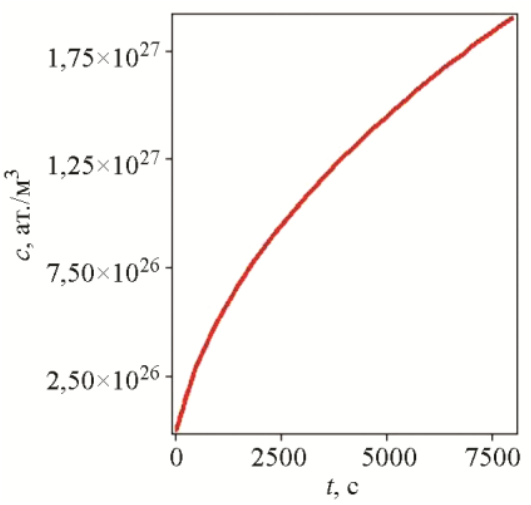

$a$

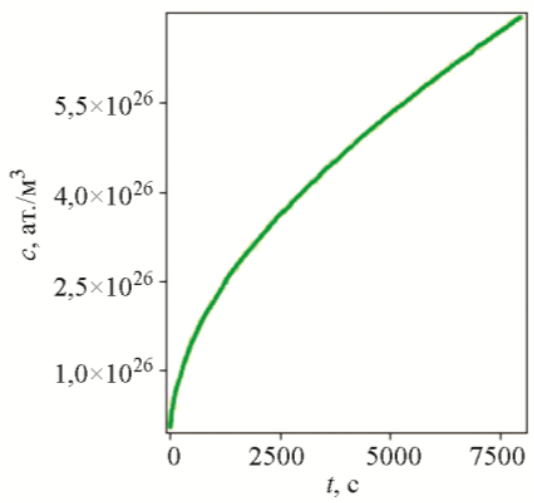

$\sigma$

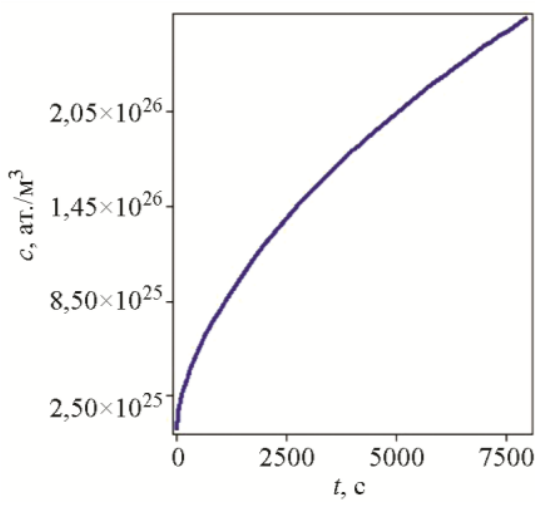

B

Рис. 3. Расчетные зависимости концентрации дейтерия от времени на глубине 3 мкм для различных значений пары коэффициентов диффузии $D_{1}$ и $D_{2}: a-D_{1}=10^{-16} \mathrm{~m}^{2} / \mathrm{c}$; $D_{2}=10^{-15} \mathrm{M}^{2} / \mathrm{c} ; \sigma-D_{1}=10^{-15} \mathrm{~m}^{2} / \mathrm{c} ; D_{2}=10^{-14} \mathrm{M}^{2} / \mathrm{c} ; \theta-D_{1}=10^{-14} \mathrm{M}^{2} / \mathrm{c} ; D_{2}=10^{-13} \mathrm{M}^{2} / \mathrm{c}$ 


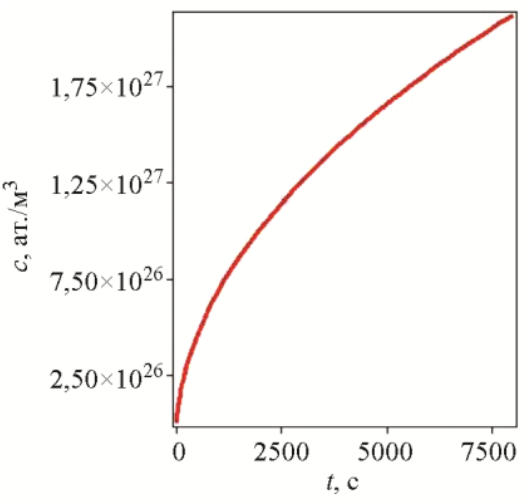

$a$

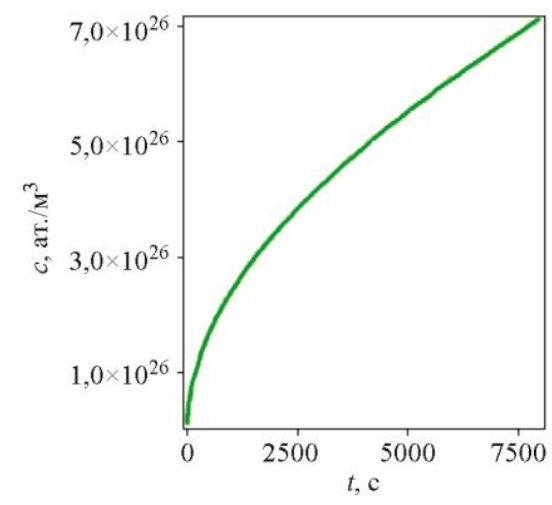

$\sigma$

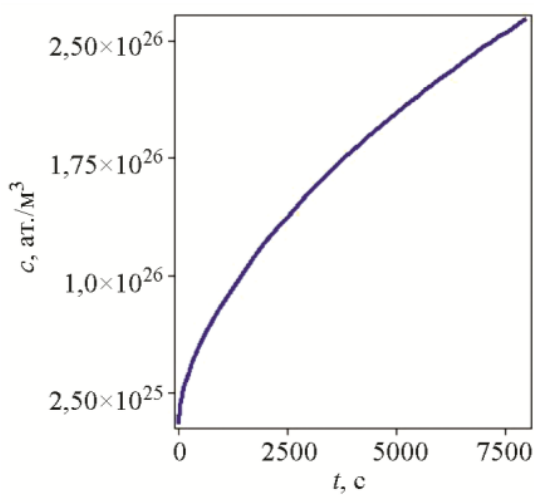

B

Рис. 4. Расчетные зависимости концентрации дейтерия от времени на глубине 3,3 мкм для различных значений пары коэффициентов диффузии $D_{1}$ и $D_{2}: a-D_{1}=10^{-16} \mathrm{~m}^{2} / \mathrm{c}$; $D_{2}=10^{-15} \mathrm{M}^{2} / \mathrm{c} ; \sigma-D_{1}=10^{-15} \mathrm{~m}^{2} / \mathrm{c} ; D_{2}=10^{-14} \mathrm{M}^{2} / \mathrm{c} ; \theta-D_{1}=10^{-14} \mathrm{M}^{2} / \mathrm{c} ; D_{2}=10^{-13} \mathrm{M}^{2} / \mathrm{c}$

\section{5. Методика и примеры расчета коэффициентов диффузии в облучаемой и необлучаемой зонах образцов}

Исследование влияния радиационных дефектов на коэффициенты диффузии дейтерия в рамках методики NRAOL выполним с использованием вычислительного алгоритма на основе метода наименьших квадратов (МНК). Для этого выполним обработку экспериментальных данных $\tilde{c}\left(x^{*}, t_{k}\right)$, полученных с помощью методики NRAOL, для концентрационного профиля дейтерия на глубине, меньшей глубины расположения источника диффузии $0<x^{*}<x_{0}$, в различные моменты времени $t_{1}<t_{2}<t_{3}<\ldots$ В качестве аппроксимирующей кривой примем аналитическое решение (15) задачи диффузии (1)-(5), а коэффициенты диффузии дейтерия в облучаемой и необлучаемой областях $D_{1}$ и $D_{2}$ будут служить параметрами метода. Тогда в соответствии с критерием МНК постоянные коэффициенты $D_{1}$ и $D_{2}$ определим из условия минимизации суммы квадратов отклонений экспериментальных значений от значений, рассчитанных по формуле (15) в заданные моменты времени $t_{k}$ :

$$
\sum_{k=1}^{n}\left(\tilde{c}\left(x^{*}, t_{k}\right)-c_{1}\left(x^{*}, t_{k}\right)\right)^{2} \rightarrow \min .
$$

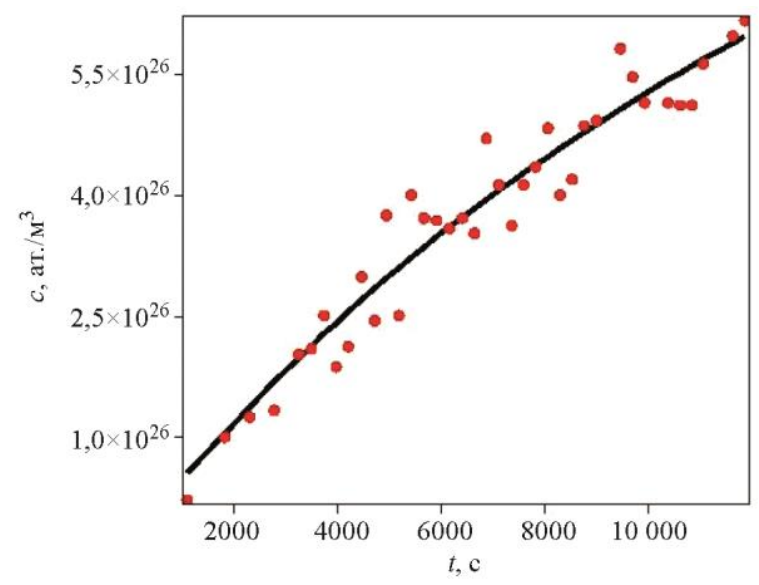

Рис. 5. Аппроксимация экспериментальных данных, полученных для никеля при $T=23{ }^{\circ} \mathrm{C}$, аналитической зависимостью (15).

Точки - экспериментальные данные, кривая - аналитическая зависимость

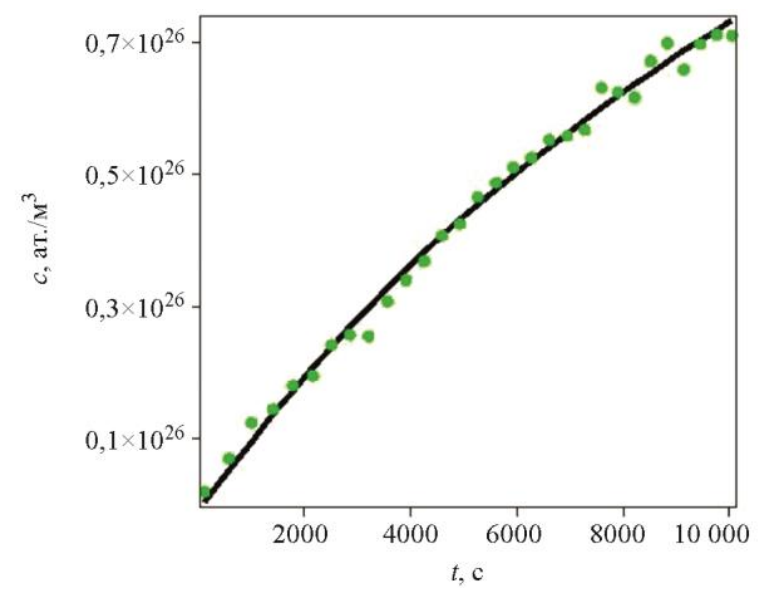

Рис. 6. Аппроксимация экспериментальных данных, полученных для натрия при $T=17^{\circ} \mathrm{C}$, аналитической зависимостью (15). Точки - экспериментальные данные, кривая - аналитическая зависимость 
Для верификации метода численные расчеты были проведены для образцов никеля и натрия с помощью компьютерной программы, созданной на базе алгоритма. Отклонения экспериментальных данных от аналитической зависимости (15) составляли 15-20 \% и соотносились со средней квадратичной погрешностью измерения концентрационных профилей.

На рис. 5 представлены результаты аппроксимации экспериментальных данных, полученных для глубины $x^{*}=1,45$ мкм во временном интервале $1107 \mathrm{c} \leq t_{k} \leq 11845$ с для образцов никеля при температуре $23{ }^{\circ} \mathrm{C}$, аналитической зависимостью (15). Вычисления проводились при $w=$ $=10^{18}\left(\mathrm{~m}^{2} \cdot \mathrm{c}\right)^{-1} ; x_{0}=3,15$ мкм. Были получены значения коэффициентов диффузии $D_{1}=2 \cdot 10^{-15} \mathrm{~m}^{2} / \mathrm{c}$; $D_{2}=2 \cdot 10^{-14} \mathrm{~m}^{2} / \mathrm{c} ; D_{2} / D_{1}=10$. Статистическая погрешность в определении $D_{1}$ и $D_{2}$ составляла $15 \%$.

На рис. 6 представлены результаты аппроксимации экспериментальных данных, полученных для глубины $x^{*}=7,8$ мкм во временном интервале $125,5 \mathrm{c} \leq t_{k} \leq 10061$ с для образцов натрия при температуре $17^{\circ} \mathrm{C}$. Параметры для расчетов: $w=4 \cdot 10^{17}\left(\mathrm{M}^{2} \cdot \mathrm{c}\right)^{-1} ; x_{0}=14,85$ мкм. Найденные значения коэффициентов диффузии: $D_{1}=7 \cdot 10^{-14} \mathrm{~m}^{2} / \mathrm{c} ; D_{2}=2 \cdot 10^{-13} \mathrm{M}^{2} / \mathrm{c} ; D_{2} / D_{1}=$ 3,5 , статистическая погрешность в определении $D_{1}$ и $D_{2}$ составляла $5 \%$.

\section{6. Заключение}

В работе была предложена и апробирована новая методика для исследования влияния точечных дефектов на коэффициенты диффузии дейтерия в металлах, конкретными объектами исследования были радиационные дефекты, сформированные при облучении образцов ускоренными дейтронами. При разработке методики было использовано упрощающее предположение, согласно которому диффузия дейтерия описывается только двумя диффузионными константами. Одна из них характеризует подвижность атомов дейтерия в образце без радиационных дефектов, а другая - в материале, содержащем дефекты. В общем случае справедливость этого предположения не очевидна. Однако можно отметить, что в конкретных примерах, использованных в работе, такие модельные представления оказались удовлетворительными. Во-первых, радиационные дефекты приводили к уменьшению коэффициентов диффузии дейтерия, что соответствует традиционным представлениям о механизме ловушек. Во-вторых, отклонения экспериментальных точек от аналитических зависимостей $c\left(x^{*}, t\right)$ не превышали статистическую погрешность ядерно-физических измерений концентрации дейтерия.

\section{Благодарность}

Работа выполнена в рамках государственного задания ФАСО России (тема «Спин» № 01201463330) и частично поддержана РФФИ (проект № 17-03-00101).

\section{Литература}

1. Ishikava T., Mclellan R. B. The low-temperature diffusion of Hydrogen through annealed, quenched and aged gold // Phys. Chem. Solids. - 1985. - Vol. 46, iss. 12. - P. 1393-1396. DOI: $10.1016 / 0022-3697(85) 90078-2$.

2. Ishikava T., Mclellan R. B. The G. P. zones in Al-Cu alloys-I // Acta Metallurgica. 1985. - Vol. 33, iss. 11. - P. 1945-1955. - DOI: 10.1016/0001-6160(85)90117-8.

3. Deuterium diffusion in proton conductors $\mathrm{La}_{0.9} \mathrm{Sr}_{0.1} \mathrm{ScO}_{3-\delta}$ and $\mathrm{BaZr}_{0.9} \mathrm{Y}_{0.1} \mathrm{O}_{3-\delta}$ at room temperature / V. B. Vykhodets, T. E. Kurennykh, O. A. Nefedova, V. P. Gorelov, A. Yu. Stroeva, V. B. Balakireva, E. V. Vykhodets, S. I. Obukhov // Solid State Ionics. - 2014. - Vol. 263. P. 152-156. - DOI: 10.1016/j.ssi.2014.06.003.

4. Vykhodets V. B., Kurennykh T. E., Nefedova O. A. Application of Nuclear Reaction Analysis to Studying Hydrogen Diffusion and Nonstoichiometry in Metal Oxides // Diagnostics, Resource and Mechanics of materials and structures. - 2015. - Iss. 5. - P. 31-44. - URL: http://dreamjournal.org/issues/2015-5/2015-5 57.html
5. $\quad$ Лыков А. В. Теория теплопроводности. - М. : Высшая школа, 1967. - 600 с.

6. Carslaw H. S., Jaeger J. C. Conduction of Heat in Solids. - London : Oxford University Press, 1959. - 510 p.- ISBN-10: 0198533039, ISBN-13: 978-0198533030.

7. Doetsch G. Anleitung zum praktischen Gebrauch der Laplace-Transformation und der Z-Transformation. - München, Wien : Oldenbourg, 1989. - 256 p. - ISBN-10: $\underline{3486213105}$, ISBN-13: 9783486213102.

8. Лобанов М. Л., Зорина М. А. Методы определения коэффициентов диффузии. Екатеринбург : Изд-во Урал. ун-та, 2017. - 100 с. - ISBN 978-5-7996-2098-1. 